\section{Die Indikation zur Kortikosteroid- Therapie der Sarkoidose und die Bewertung ihrer Resultate}

Seit der Einführung der Kortikosteroid-Therapie der Sarkoidose besteht Uneinigkeit über die Indikation und die Einschätzung der Resultate. Weitgehend Einigkeit besteht darüber, dass eine nicht-symptomatische Sarkoidose keiner Therapie bedarf, dass beim Löfgren-Syndrom eine Therapie mit nichtsteroidalen Antiphlogistika ausreicht und dass bei symptomatischer Beteiligung von ZNS, Auge, Herz oder Niere oder bei Hyperkalzämie eine Kortikosteroid-Therapie indiziert ist [19].

Problematisch ist die Indikationsstellung bei einer diskreten Symptomatik mit Husten oder Dyspnoe, bei schwer fassbaren Befindlichkeitsstörungen wie chronischer Müdigkeit und Muskelschmerzen oder bei einem progredienten radiologischen Befund ohne oder nur mit diskreter Symptomatik. Auch in Fällen mit einer retikulo-nodulären Zeichnungsvermehrung im Thorax-Röntgenbild ohne Lymphadenopathie (Тур III), die häufig chronifizieren, kommt es in etwa 35\% noch zu einer Spontanremission mit normalem Thorax-Röntgenbild. Bei den radiologischen Typen I (bihiläre Lymphadenopathie) und II (bihiläre Lymphadenopathie mit retikulo-nodulärer Zeichnungsvermehrung) sind Spontanremissionen mit Normalisierung des Thorax-Röntgenbildes in 80 bzw. 50\% innerhalb von 3 Jahren zu erwarten [10]. Daher ist eine Beobachtungsphase von 6 Monaten bei diesen Patienten anzuraten, um abzuwarten, ob eine Spontanremission eintritt.

Ist dies nicht der Fall, so kann man meist einen sich entwickelnden Organschaden oder eine eindeutig bestehende Symptomatik dokumentieren, dessen Rückbildung bzw. Sistieren das Therapieziel darstellt. Eine aussagekräftige dänische Studie konnte zeigen, dass eine dokumentierte aber symptomfreie Sarkoidose eine gute Prognose hat [11]. Laborparameter können die entzündliche Aktivität der Sarkoidose erfassen. Für die Indikation oder das Beenden der Therapie können sie jedoch nur stützende Befunde liefern [12]. Arzt und Patient sollten daher vorab ein Ziel der KortikosteroidTherapie formulieren. Ist dieses Ziel erreicht, so kann die Therapie beendet werden. Lässt sich das Ziel mit akzeptablen Kortikosteroid-Dosierungen in einem Zeitraum von 6-12 Monaten nicht erreichen, so besteht eine Kortikosteroidresistente Sarkoidose, die mit anderen Modalitäten behandelt werden muss, da eine Langzeittherapie mit Kortikosteroiden ein zu ungünstiges Verhältnis von Nebenwirkungen zu Risi-

Pneumologie 2000; 54: 284-286

(C) Georg Thieme Verlag Stuttgart · New York ISSN 0934-8387
J. Müller-Quernheim

Medizinische Klinik, Forschungszentrum Borstel ken aufweist. Ein vorab formuliertes Therapieziel, das anhand von Befunden überprüfbar ist, verhindert, dass eine Entscheidung über die weitere Vorgehensweise verschleppt wird und unbeabsichtigt eine in ihrer Effektivität nicht überprüfbare Kortikosteroid-Langzeittherapie resultiert.

Üblicher Weise wird die Therapie mit 0,6 bis 0,8 mg Prednisolon pro kg Körpergewicht und Tag begonnen. In Abhängigkeit vom Ansprechverhalten der Erkrankung wird die schrittweise Dosisreduktion nach 2 bis 6 Wochen begonnen und die Schritte werden so gewählt, dass die Therapie nach etwa 6 Monaten beendet ist. Es existieren keine kontrollierten Studien zur Startdosis, Gesamtdosis oder Therapiedauer. Diese Vorgehensweise beruht auf dem Erfahrungsschatz seit der Einführung dieser Therapieform.

Eine Reihe von älteren, teilweise recht großen Studien haben sich mit den Resultaten der Kortikosteroid-Therapie beschäftigt und kamen zu dem Schluss, dass eine KortikosteroidTherapie nicht gerechtfertigt ist, da behandelte und unbehandelte Kollektive sich in ihren Langzeitergebnissen nicht unterscheiden (zwei Beispiele: [13,14]). In der Mehrzahl dieser Studien war der radiologische Typ sowohl die Indikation für die Kortikosteroid-Therapie als auch der Zielparameter. Ein Therapieziel, wie oben gefordert, mag bei einzelnen Patienten vorgelegen haben, ging aber in die Auswertung nicht ein. Die Rückbildung einer Restriktion und der Symptomatik, ein Sistieren des Hustens oder eine verbesserte körperliche Belastbarkeit wurden nicht erfasst. Gerade diese Parameter sind jedoch funktionell und für die Lebensqualität des Patienten wichtig und es ist zwischenzeitlich gut dokumentiert, dass die entzündliche, therapiebedürftige Aktivität der Sarkoidose mit den radiologischen Typen in keiner Weise korreliert $[8,15,16]$. Bereits Louis Siltzbach, einem der ersten Befürworter der Kortikosteroid-Therapie der Sarkoidose, wurde vorgehalten, dass sich die radiologischen Typen bei seinen Patienten-Kohorten nicht änderten. Er hat erwidert, dass es seinen Patienten unter und nach der Therapie besser ging und dass dies für ihn ein größeres Gewicht als ein radiologischer Befund hat.

Heute kennen wir Details der Entzündungsvorgänge bei Sarkoidose und wissen, dass von aktivierten Leukozyten inflammatorische Prozesse unterhalten werden, die einerseits einen Funktionsverlust der betroffenen Organe und andererseits die den Patienten belästigende Symptomatik hervorrufen. Mediatoren wie Interleukin 2, Interferon- $\gamma$ und Tumornekrosefaktor- $\alpha$ stellen zentrale Mechanismen dieser Vorgänge dar [16]. Bei der Mehrzahl der Patienten lässt sich beides 
durch Kortikosteroide supprimieren und eine langanhaltende Remission herbeiführen [17-19]. Eine Suppression der Entzündungsmediatoren geht mit dem Eintreten einer Remission parallel und eine erneute Aktivität der Leukozyten ist meist mit einem Rezidiv vergesellschaftet. Der radiologische Befund reagiert nicht oder nur sehr verzögert auf diese immunologischen Veränderungen, so dass er zur frühen Indikationsstellung nicht mehr oder nur in Ausnahmefällen herangezogen werden kann $[15,20]$.

In den letzten Jahren haben sich zwei größere Studien mit der Kortikosteroid-Therapie der Sarkoidose beschäftigt. G. Hunninghake et al. analysierten Patienten-Gruppen, die nach einer Beobachtungsphase mit Dokumentation eines pulmonalen Funktionsverlustes mit Kortikosteroiden therapiert wurden oder im Verlauf beobachtet wurden, wenn kein progredienter Funktionsschaden erkennbar war. In der Therapiegruppe besserte sich die Lungenfunktion bei 16 und stabilisierte sich bei 20 von 36 Patienten. In der Beobachtungsgruppe kam es nur bei 8 von 55 zu einem Funktionsverlust der Lunge, der sich bei 6 durch eine KortikosteroidTherapie und die Behandlung von Begleit- und Folgeerkrankungen aufhalten ließ. Die Nachbeobachtung zeigte, dass in der Behandlungsgruppe etwa $14 \%$ ein Rezidiv nach Therapieende erlitten. Bei der beschriebenen Vorgehensweise zur Indikationsstellung kann somit ein Kollektiv identifiziert werden, das von einer Kortikosteroid-Therapie der Sarkoidose eindeutig profitiert [21].

Eine große multizentrische Studie der British Thoracic Society teilte Patienten mit persistierendem, radiologischem Befund in eine Gruppe $(n=27)$ mit Langzeit-Kortisontherapie (mindestens 18 Monate) und in eine Gruppe $(n=31)$ mit Verlaufsbeobachtung und Therapie (6-9 Monate) nur bei Symptomatik. In der Langzeitgruppe musste die Therapie zweimal wegen Kortikosteroid-Nebenwirkungen abgebrochen werden. In der Beobachtungsgruppe kam es lediglich bei 6 Patienten zu einer Symptomatik, die eine Therapie erforderte. Nach vier Jahren unterschieden sich die beiden Gruppen kaum. Die Autoren bewerteten eine Reihe von klinischen Parametern mit Punkten und fanden einen signifikanten Vorteil für die Langzeittherapiegruppe. Für die Dyspnoe und den radiologischen Befund fanden sich jedoch keine signifikanten Unterschiede. Die Autoren empfehlen zwar die Langzeittherapie, aber ihre detailreiche Darstellung der Studie erlaubt dem Leser eine eigene Urteilsbildung, und ich kann der Empfehlung der Autoren nicht folgen [22]. Nach meiner Einschätzung rechtfertigt das Ergebnis nicht den therapeutischen Aufwand, das Nebenwirkungsrisiko und die Einschränkung der Lebensqualität der Patienten. Den Autoren ist für die klare Beschreibung der vielen Aspekte der Studie zu danken, was die persönliche Einschätzung erst ermöglicht.

Eine andere Studie darf in diesem Zusammenhang nicht unerwähnt bleiben. Gottlieb et al. zeigten, dass chronisch behandlungsbedürftige Sarkoidoseverläufe mit mehreren Rezidiven vor allem in der Gruppe von Patienten zu finden waren, die bereits bei Diagnosestellung mit Kortikosteroiden behandelt werden mussten [15]. Die Autoren diskutieren die Möglichkeit, dass die initiale Kortikosteroid-Therapie die Rezidive herbeigeführt hat. Im Kontext der Erkenntnisse zur Immunpathogenese der Sarkoidose kann ich dieser Schlussfolgerung nicht zustimmen und sehe hier - wie auch die
Autoren - keine generelle Kontraindikation zur Kortikosteroid-Therapie, die sich aus dieser Verlaufsbeobachtung ableiten ließe. Insbesondere kann die Studie nicht dazu herangezogen werden, die Kortikosteroid-Therapie für ineffektiv zu erklären, da Kortikosteroid-Wirkungen auf zentrale immunpathogenetische Mechanismen zahlreich dokumentiert sind. Eine kausale Therapie stellt die Kortikosteroid-Therapie jedoch nicht dar und die Mechanismen, die zur Remission führen, sind weiterhin unbekannt. Es ist zu vermuten, dass die Kortikosteroide immunologische Regulationsmechanismen, die auch bei der Spontanremission eine Rolle spielen, begünstigen [23].

Für kortikosteroidresistente Fälle, die immerhin zwischen 10 und $20 \%$ der Patienten mit Behandlungsindikation ausmachen, stehen keine monotherapeutischen Alternativen zur Verfügung. Versuche, die Sarkoidose mit Cyclosporin A, Azathioprin, Methotrexat oder Cyclophosphamid zu therapieren, haben zwar in Einzelfällen dokumentierte Erfolge gezeigt, aber Parameter, die einen Behandlungserfolg voraussagen, konnten nicht herausgearbeitet werden, so dass Subkollektive, die von derartigen Therapiealternativen vorhersehbar profitieren, nicht identifiziert werden können [24-29]. Literatur, die Erfolge von Vorgehensweisen außerhalb der Schulmedizin, wie Eigenblutbehandlung, über erweiterte Fallberichte hinaus dokumentiert, existiert nicht. Immunsuppressive Kombinationstherapien, die es ermöglichen, die Kortikosteroid-Dosis zu reduzieren, stehen jedoch zur Verfügung und ihre Erfolge sind ermutigend [30]. Welcher Partner bei geringster Nebenwirkungsrate und maximalem Effekt eine Kortisoneinsparung ermöglicht, ist noch offen. Eine Reihe von Kandidaten wie Azathioprin, Methotrexat, Pentoxifyllin und Thalidomid sind in Erprobung [30-33].

Solange eine ätiologische Therapie der Sarkoidose nicht möglich ist, muss bei persistierenden Symptomen und/oder progredientem Funktionsverlust eines befallenen Organs die Kortikosteroid-Therapie als Stand der Kunst angesehen werden, denn die Literatur belegt überzeugend, dass diese Therapie lang anhaltende Remissionen induziert. Da ein formaler Beweis in Form von doppelblinden, plazebokontrollierten Studien nicht führbar ist, muss die Indikation auf der Basis des vorliegenden Erfahrungsschatzes gestellt werden und hier ist die Kortikosteroid-Therapie die Option der ersten Wahl.

\section{Literatur}

${ }^{1}$ duBois RM. Corticosteriods in sarcoidosis: friend or foe? Eur Respir J 1994; 7: 1203 - 1209

2 Sharma O. Pulmonary sarcoidosis and corticosteroids. Am Rev Respir Dis 1993; 147: 1598 - 1600

${ }^{3}$ Endres P, Müller-Quernheim J. Alveolitiden und granulomatöse Lungenerkrankungen. In: Ferlinz R, ed. Pneumologie in Klinik und Praxis. Stuttgart: Thieme, 1994: 487-540

${ }^{4}$ Schaberg T, Schonfeld N, Loddenkemper R, Lode H. Therapie der Sarkoidose. Dtsch Med Wochenschr 1995; 120 (20): 725-727

${ }^{5}$ Müller-Quernheim J, Dalhoff K, Schnabel A. Immunsuppressive Therapie bei interstitiellen Lungenerkrankungen und immunologisch vermittelten Pneumonitiden. Internist (Berl) 1997; 38 (6): $553-561$

${ }^{6}$ Deutsche Gesellschaft für Pneumologie. Empfehlungen zur Diagnostik und Therapie der Sarkoidose. Pneumologie 1998; 52 (1): $26-30$ 
${ }^{7}$ Lynch JPR. Pulmonary sarcoidosis: current concepts \& controversies. Compr Ther 1997; 23 (3): 197-210

${ }^{8}$ Judson MA. An approach to the treatment of pulmonary sarcoidosis with corticosteroids: the six phases of treatment. Chest 1999; 115 (4): $1158-1165$

${ }^{9}$ DeRemee R. The present status of treatment of pulmonary sarcoidosis: A house divided. Chest 1977; 71: 388 - 393

${ }^{10}$ Hillerdal G, Nöu E, Osterman K, Schmekel B. Sarcoidosis: epidemiology and prognosis. A 15-year European study. Am Rev Respir Dis 1984; 130: 29-32

11 Vestbo J, Viskum K. Respiratory symptoms at presentation and long-term vital prognosis in patients with pulmonary sarcoidosis. Sarcoidosis 1994; 11: 123-125

12 Müller-Quernheim J. Serum markers for the staging of disease activity of sarcoidosis and other interstitial lung diseases of unknown etiology. Sarcoidosis Vasc Diffuse Lung Dis 1998; 15 (1): $22-37$

${ }^{13}$ Israel H, Fouts D, Beggs R. A controlled trial of prednisone treatment of sarcoidosis. Am Rev Respir Dis 1973; 107: 609-614

${ }^{14}$ Zaki MH, Lyons HA, Leilop L, Huang CT. Corticosteroid therapy in sarcoidosis. A five-year, controlled follow-up study. N Y State J Med 1987; 87: 496-499

${ }^{15}$ Gottlieb JE, Israel HL, Steiner RM, Triolo J, Patrick H. Outcome in sarcoidosis. The relationship of relapse to corticosteroid therapy. Chest 1997; 111: 623-631

${ }^{16}$ Müller-Quernheim J. Sarcoidosis: immunopathogenetic concepts and their clinical application. Eur Respir J 1998; 12 (3): $716-738$

17 Pinkston P, Saltini C, Müller-Quernheim J, Crystal RG. Corticosteroid therapy suppresses spontaneous interleukin-2 release and spontaneous proliferation of lung T-lymphocytes of patients with active pulmonary sarcoidosis. J Immunol 1987; 139: 755 760

${ }^{18}$ Baughman RP, Strohofer SA, Buchsbaum J, Lower EE. Release of tumor necrosis factor by alveolar macrophages of patients with sarcoidosis. J Lab Clin Med 1990; 115: 36-42

${ }^{19}$ Strausz J, Männel D, Pfeifer S, Borkowski A, Ferlinz R, MüllerQuernheim J. Spontaneous monokine release by alveolar macrophages in chronic sarcoidosis. Int Archs Allergy appl Immun 1991; 96: 68 - 75

${ }^{20}$ Baumann MH, Strange C, Sahn SA. Do chest radiographic findings reflect the clinical course of patients with sarcoidosis during corticosteroid withdrawal? AJR Am J Roentgenol 1990; 154 (3): $481-485$

${ }^{21}$ Hunninghake GW, Gilbert S, Pueringer R et al. Outcome of the treatment of sarcoidosis. Am J Respir Crit Care Med 1994; 149 : $893-898$

${ }^{22}$ Gibson G, Prescott R, Muers M et al. British Thoracic Society sarcoidosis study: Effects of long term corticosteroid treatment. Thorax 1996; 51: $238-247$

${ }^{23}$ Zissel G, Homolka J, Schlaak J, Schlaak M, Müller-Quernheim J. Anti-inflammatory cytokine release by alveolar macrophages in pulmonary sarcoidosis. Am J Respir Crit Care Med 1996; 154: $713-719$

${ }^{24}$ Lynch III JP, McCune WJ. Immunosuppressive and cytotoxic pharmacotherapy for pulmonary disorders. Am J Respir Crit Care Med 1997; 155: $395-420$

25 Pacheco Y, Marechal C, Marechal F, Biot N, Perrin-Fayolle M. Azathioprine treatment of chronic pulmonary sarcoidosis. Sarcoidosis 1985; 2: 107-113

${ }^{26}$ Hammond J, Bateman E. Successful treatment of life-threatening steroid-resistant pulmonary sarcoidosis with cyclosporin in a patient with systemic lupus erythematosus. Respir Med 1990; 84: $77-80$

${ }^{27}$ Martinet Y, Pinkston P, Saltini C, Spurzem J, Müller-Quernheim J, Crystal RG. Evaluation of the in vitro and in vivo effects of cyclosporine on the T-lymphocyte alveolitis of active pulmonary sarcoidosis. Am Rev Respir Dis 1988; 138: 1242 - 1248

${ }^{28}$ Lower EE, Broderick JP, Brott TG, Baughman RP. Diagnosis and management of neurological sarcoidosis. Arch Intern Med 1997; 157 (16): $1864-1868$

${ }^{29}$ Lower EE, Baughman RP. The use of low dose methotrexate in refractory sarcoidosis. Am J Med Sci 1990; 299 (3): 153-157

${ }^{30}$ Müller-Quernheim J, Kienast K, Held M, Pfeifer S, Costabel U. Treatment of chronic sarcoidosis with an azathioprine/prednisolone regimen. Eur Respir J 1999; 14 (5): 1117- 1122

${ }^{31}$ Zabel P, Entzian P, Dalhoff K, Schlaak M. Pentoxifylline in treatment of sarcoidosis. Am J Respir Crit Care Med 1997; 155: 1665-1669

32 Baughman RP, Lower EE. Steroid-sparing alternative treatments for sarcoidosis. Clin Chest Med 1997; 18 (4): 853-864

${ }^{33}$ Carlesimo M, Giustini S, Rossi A, Bonaccorsi P, Calvieri S. Treatment of cutaneous and pulmonary sarcoidosis with thalidomide. J Am Acad Dermatol 1995; 32 (5 Pt 2): 866-869

Prof. Dr. J. Müller-Quernheim

Medizinische Klinik

Forschungszentrum Borstel

Parkallee 35

23845 Borstel

E-mail: jmq@fz-borstel.de 